\title{
La selección modal en las cláusulas adverbiales introducidas por como si
}

\author{
The modal selection in the adverbial clauses \\ introduced by como si
}

\author{
Wiaczesław Nowikow \\ Uniwersytet Łódzki \\ nowikow_2000@yahoo.com.mx
}

\begin{abstract}
The conjunction como si select usually in the subordinated adverbial clauses (modal, conditional and comparative) the tenses of the subjunctive mood cantara / cantase and hubiera / hubiese cantado. However in some papers is mentioned the possibility of the construction of como si with the tenses of indicative mood. This paper contains the analysis of the modal selection after como si and, particularly, the possible substitution of subjunctive by the indicative tenses. The analyses is realized on the ground of the corpus of Real Academia Española (CORPES XXI, CREA, CREA. Versión anotada).
\end{abstract}

Keywords: subordinadas adverbiales, como si, selección modal, modo indicativo, modo subjuntivo, subordinated adverbial clauses, modal selection, indicative mood, subjunctive mood

\section{EL ESTATUS GRAMATICAL DE LAS SUBORDINADAS CON COMO SI}

En el caso de las construcciones con el nexo como si se plantean, básicamente, dos tipos de problemas. La primera cuestión se refiere a la clasificación sintácticosemántica. Desde esta perspectiva, la conjunción subordinante como si se considera mayoritariamente como introductor de subordinadas modales o condicionales. Por ejemplo, en la $N G L E$ (2010: 1942) y en Veiga \& Mosteiro Louzao (2006: 200-203) como si se encuentra en los párrafos dedicados a las condicionales. Por su parte, en Alarcos Llorach (1994: 363) y en Gutiérrez Araus (2004: 264-267) este nexo se ve incluido en las adverbiales de modo, aunque la última autora advierte que "el valor 
de como si es mitad modal, mitad condicional" (Gutiérrez Araus 2004: 265). Montolío (1999: 3679) llama la atención sobre el hecho de que como introduce también el significado comparativo señalando, sin embargo, que el sentido global de la construcción con como si es de tipo modal (fijémonos que estas observaciones aparecen en el capítulo dedicado a las construcciones condicionales). En cambio, Pérez Saldanya (1999: 3316) incluye como si en "otras oraciones subordinadas adverbiales" señalando, no obstante, que "por el hecho de formarse a partir de la conjunción si presenta unas peculiaridades hasta cierto punto semejantes a las de las oraciones condicionales" (Pérez Saldanya 1999: 3317).

El segundo problema se ve relacionado con la selección de los modos verbales. Como es sabido, en español como si se construye, habitualmente, con los tiempos del modo subjuntivo: copretérito o imperfecto cantase / cantara y antecopretérito o pluscuamperfecto hubiese / hubiera cantado. Así, según Alarcos Llorach (1994: 363), la construcción con "como si (seguida, claro es, de subjuntivo) origina el mismo efecto de sentido que como que y es hoy más frecuente". De modo que Alarcos Llorach subraya la naturalidad de la combinación de como si con las formas subjuntivas, lo que no debe sorprender si tomamos en consideración que se trata de acciones caracterizadas por no-aserción en cuyo caso el compromiso con la verdad $\mathrm{y}$, por lo tanto, la factualidad son prácticamente nulos:

(1) Me trata como si no nos conociésemos \{sí que nos conocemos\}.

(2) Nos saludamos como si no nos hubiéramos visto nunca \{sí que nos habíamos visto\}.

Sin embargo, como apuntan algunos autores, en el español se dan los empleos donde el nexo como si se construye con los tiempos del modo indicativo, en primer lugar con el presente canta y con el copretérito (imperfecto) cantaba:

(3) Es como si yo te digo que me voy y que ya... (Pérez Saldanya 1999: 3317).

(4) - No volveré hasta el lunes.

- Por mi, como si no vuelves en un mes (Montolío 1999: 3681).

(5) Este chico estudia como si es un superdotado (Gutiérrez Araus 2004: 267).

(6) Por mí, como si te ibas ahora mismo (Pérez Saldanya 1999: 3317).

(7) Trabajaba como si no sabía hacer otra cosa (Gutiérrez Araus 2004: 267).

Según los autores citados supra, en tales casos, se observa cierta afinidad entre las construcciones como $s i+$ indicativo y las cláusulas condicionantes reales $(s i+c a n t a)$ y no reales $(s i+$ cantase / cantara $)$. Esto se refiere, en primer lugar, a las combinaciones de como si con el presente de indicativo (véase el ejemplo 3), pero también, en parte, a las construcciones de como si con cantaba (véase el ejemplo 7). Podríamos observar, con este motivo, que en dichas distribuciones la conjunción como, hasta cierto punto, se independiza uniéndose si más al verbo y asemejándose, de esta manera, a las cláusulas con valor condicionante. Por otro lado, algunos autores (Montolío 1999: 3681; Pérez Saldanya 1999: 3317) llaman la atención sobre el 
carácter enfático y replicativo de la construcción como $s i+$ indicativo, lo que se manifiesta en los estilos coloquial e informal del español actual (véanse los ejemplos 4 y 6$)$.

A primera vista, los usos de indicativo después de como si parecen poco frecuentes y provocan dudas de naturaleza normativa. Por otro lado, en los trabajos de los autores que mencionan este tipo de construcciones (véase supra) no hemos encontrado comentarios sobre la real difusión del fenómeno en cuestión. Esto nos motivó llevar a cabo, a título de prueba, una pequeña investigación con el fin de comprobar las posibilidades combinatorias del nexo como si con el modo indicativo.

\section{COMO SI+CANTABA: LOS RESULTADOS DEL ANÁLISIS DEL MATERIAL DEL CORPUS}

Hemos decidido examinar las concordancias de la conjunción como si con veinte verbos de alta frecuencia de uso, a saber: dar, decir, estar, haber, hacer, ir, pedir, pensar, poder, poner, querer, saber, salir, sentir, ser, tener, trabajar, traer, venir, ver. También hemos optado por comprobar dichos verbos en forma de copretérito (imperfecto) de indicativo ( $1^{\mathrm{a}}$ y $2^{\mathrm{a}}$ personas del singular), puesto que se trata de un tiempo que, dentro de los tiempos de indicativo, demuestra mayores posibilidades de modalizaciones contrafactuales a través de desplazamientos temporales de tipo De buena gana me tomaba un mosto (en este momento no lo estoy tomando) o Si estuviese libre, te ayudaba a preparar la comida (en este momento no te ayudo: estoy ocupado) (véase, a este respecto, p. ej., Nowikow 2017a: 147-148). Además, es el tiempo que al desempeñar varias funciones estilístico-discursivosituacionales, con frecuencia transmite contenidos matizados de validez limitada (p. ej., los llamados valores de cortesía, de renarración o lúdico; véase Nowikow 2017a: 152-156). Tanto los valores contrafactuales dislocados como los de validez limitada, hasta cierto punto, coinciden, a nivel nocional, con el contenido funcional de [no-aserción] propio del modo subjuntivo. El material analizado en este estudio proviene de los corpus de la RAE:

1) CORPES XXI (REAL ACADEMIA ESPAÑOLA: Banco de datos (CORPES XXI) [en línea]. Corpus del Español del Siglo XXI (CORPES), $<$ http://www.rae.es $>$ [fecha de la consulta: 24.11.2018]),

2) CREA (REAL ACADEMIA ESPAÑOLA: Banco de datos (CREA) [en línea]. Corpus de referencia del español actual, <http://www.rae.es $>$ [fecha de la consulta: 29.11.2018]) y

3) CREA. Versión anotada (REAL ACADEMIA ESPAÑOLA: Banco de datos (CREA. Versión anotada) [en línea]. Corpus de referencia del español actual, $<$ http://www.rae.es $>$ [fecha de la consulta: 29.11.2018]). 
En el caso del CORPES XXI hemos utilizado la versión provisional 0.9, de 10 de julio de 2018. Se trata de un recurso en construcción que engloba casi 277 millones de formas procedentes de los textos desde el año 2001 en adelante.

En cambio, la última versión del CREA (3.2, junio de 2008) contiene más de 160 millones de formas desde el año 1975 y hasta el año 2004. Sin embargo, su manera de presentar las concordancias y las estadísticas no coincide con la de CORPES XXI.

Por lo tanto, hemos recurrido también a la versión anotada del CREA (versión 0.1 de 2015) que tiene el mismo sistema de codificación que el CORPES XXI, es decir, permite consultar por lemas, formas y categorías gramaticales $\mathrm{y}$, cosa quizá más importante en este caso, posee el mismo modo de anotación de las concordancias y de las estadísticas. La frontera cronológica de la versión anotada del CREA es el año 2000 que justamente precede el primer año de los datos aportados por el CORPES XXI (2001). Por otro lado, cabe señalar que la versión anotada del CREA no contiene textos orales. Los incluye, en cambio, la versión no-anotada del CREA.

La cantidad y la repartición de las formas de indicativo con como si registradas en nuestro corpus se presentan de la siguiente manera:

\begin{tabular}{|l|c|c|c|}
\hline \multicolumn{1}{|c|}{ VERBO } & CORPES XXI & CREA & CREA versión anotada \\
\hline Dar & 0 & 0 & 0 \\
\hline Decir & 0 & 0 & 0 \\
\hline Estar & 2 & 0 & 0 \\
\hline Haber & 13 & 0 & 2 \\
\hline Hacer & 5 & 0 & 0 \\
\hline Ir & 1 & 1 & 0 \\
\hline Pedir & 0 & 0 & 0 \\
\hline Pensar & 0 & 0 & 0 \\
\hline Poder & 2 & 0 & 0 \\
\hline Poner & 0 & 0 & 0 \\
\hline Querer & 0 & 0 & 0 \\
\hline Saber & 0 & 0 & 0 \\
\hline Salir & 0 & 0 & 0 \\
\hline Sentir & 0 & 0 & 0 \\
\hline Ser & 11 & 2 & 1 \\
\hline Tener & 1 & 0 & 0 \\
\hline Trabajar & 0 & 1 & 0 \\
\hline Traer & 0 & 0 & 0 \\
\hline Venir & 0 & 0 & 0 \\
\hline Ver & 0 & 0 & 5 \\
\hline & 35 & 4 & \\
\hline
\end{tabular}


Como se ve, predominan, con gran ventaja, las formas procedentes del CORPES XXI: 35, seguidas por las de la versión anotada del CREA (5) y por las del CREA (4).

El análisis nos ha permitido destacar las siguientes características más generales del material examinado:

1) La forma cantaba se da con 8 verbos: estar, haber, hacer, ir, poder, ser, tener, trabajar. Esto quiere decir que el indicativo no aparece en la mayoría de los casos (12 verbos). Cabe señalar que, al parecer, se trata de los verbos más frecuentes caracterizados, además, por gran irregularidad morfológica. Por lo tanto, podríamos constatar que esta última se ve acompañada por otro tipo de irregularidad, esta vez de índole morfosintáctica: la construcción con la conjunción subordinante con como si.

2) Por otro lado, llama la atención el hecho de que los empleos en cuestión se concentren en tres verbos: haber, hacer, ser. De la cantidad global de 44 casos 15 usos fueron registrados con el verbo haber, 7 con hacer y 13 con ser, lo que quiere decir que las construcciones con los tres verbos señalados constituyen un $80 \%$ del total (35 de 44). Los 6 verbos restantes se construyen con el indicativo muy esporádicamente: estar (2), ir (2), poder (2), tener (2) y trabajar (1). Esto significa que el alcance de la repartición distribucional de como si + cantaba se ve muy limitado.

3) No todos los empleos son estrictamente del copretérito. De 15 casos del verbo haber (13 en el CORPES XXI y 2 en la versión anotada del CREA) 14 se dan con la forma de antepréterito (pluscuamperfecto) de tipo había cantado ${ }^{1}$. Solo 1 caso (CORPES XXI) representa el copretérito:

(8) Como si había veinte, no lo creo, con sinceridad... (CORPES XXI, Esp.).

Además, cabe tener en cuenta que no en todos los ejemplos había cantado sustituye a hubiese / hubiera cantado. De hecho, se trata de dos estructuras diferentes. En 6 casos como si es conjunción subordinante y había cantado efectivamente reemplaza hubiese / hubiera cantado, por ejemplo:

(9) ...descubierto algo nuevo y valioso! ¡Como si había encontrado su propia identidad! o jcomo si había encontrado la razón de su existir! (CORPES XXI, El Salv.).

En cambio, en 5 casos el nexo como sí se compone de la conjunción subordinante como y adverbio afirmativo sí estableciéndose el valor comparativo entre las dos cláusulas interordinadas:

\footnotetext{
${ }^{1}$ En la versión actualizada del CORPES XXI (0.91) que apareció en enero de 2019 las formas compuestas de tipo había cantado fueron eliminadas, salvo el primer ejemplo procedente de una novela mexicana de 2006: ...mezcla de vergüenza y desolación que sufría me llenaba de preguntas sin respuesta, como si había o no traicionado a Marisol. Notemos que en esta oración había traicionado no sustituye las formas subjuntivas hubiera / hubiese traicionado matizadas las conjunciones como y si de comparación e interrogación.
} 
(10) A Hugo no le había contratado Ancona, como si había hecho antes con el propio Lino... (CORPES XXI, Esp.).

(11) Ahora estaba clarísimo, pero no sentí, como sí había sentido al conocer a Glenda... (CORPES XXI, Esp.).

Cabe señalar que incluso cuando el nexo se compone de conjunciones subordinantes como y si, estas pueden, hasta cierto punto, independizarse adquiriendo como el valor comparativo y si el condicional o el interrogativo. Esto ocurre con frecuencia en las estructuras de tipo tanto si... como si o tal / tan... como si:

(12) ...consecuencias de aquel suceso inesperado: tanto si él había cometido una imprudencia como si había sido víctima de un engaño... (CREA. Versión anotada, Esp.).

(13) De esa guisa tuvo que atender a la prensa, que le preguntó cosas tan inteligentes como si había aprovechado para leer algo mientras... (CORPES XXI, Esp.).

De modo que un $60 \%$ de los ejemplos con había cantado (9 de 14) no representa la sustitución del subjuntivo por el indicativo (con respecto a este último fenómeno, véase también infra el párrafo sobre la repartición sintáctico-semántica).

Además, creemos oportuno considerar el material examinado en este estudio desde el punto de vista de tres reparticiones: diatópica, diafásica y sintáctico-semántica.

\section{LAS REPARTICIONES DIATÓPICA Y DIAFÁSICA}

Se trata de dos reparticiones de carácter más bien externo aunque, por supuesto, importantes para la característica global del fenómeno.

Con respecto a la repartición diatópica, cabe señalar que el corpus que hemos analizado abarca 11 países: España (17 formas), El Salvador (7), México (4), Nicaragua (4), Argentina (3), Guatemala (2), Uruguay (2), Venezuela (2), Bolivia (1), Colombia (1) y Chile (1). Aunque nos damos cuenta de la cantidad reducida de las formas en cuestión, es de notar que destacan dos países: España y El Salvador con 17 y 7 casos de construcción como si + IND. Sin entrar en los problemas relacionados con las propiedades sintáctico-semánticas (véase infra), subrayemos que las formas procedentes de estos dos países rebasan la mitad del total de los ejemplos registrados (24 de 44). Al aplicar la distribución por zonas, que se encuentra en las estadísticas del CORPES XXI, resulta que predominan España (17) y México y Centroamérica (17: El Salvador 7, México 4, Nicaragua 4, Guatemala 2) al constituir un 75\% del total (34 de 44). El tercer lugar lo ocupa la zona del Rio de la Plata con 5 formas (Argentina 3 y Uruguay 2).

En cambio, de acuerdo con la repartición diafásica pueden ser distinguidas, grosso modo, dos clases: la de ficción y la de no ficción. La primera abarca, fun- 
damentalmente, novelas, relatos y teatro mientras que la segunda incluye todo tipo de textos periodísticos, es decir, reportajes, entrevistas, etc. Hay solo un caso de discurso oral grabado que fue documentado en la radio española en la entrevista con el vicepresidente de la Asamblea Permanente de Derechos Humanos de Bolivia:

(14) Bolivia // ellos nos comentaban apenas comenzaban las movilizaciones nos comentaban cómo / si estaba la sensación de / los soldados / la policía... (CORPES XXI, Esp.)

donde, sin embargo, la forma estaba, empleada en un contexto más bien afirmativo, no es sustituto del subjuntivo.

Además, hay dos ejemplos procedentes del estilo oral (entrevistas de cara a cara) de Venezuela que según CREA, se caracterizan por baja formalidad:

(15) Para mi ir para el colegio era una odisea. Imagínate tú, este, de La Campiña a Tienda Honda eso era asi una mi mamá me daba la bendición, y me daban así, tú sabes, como si iba para el África Oriental... (CREA, Ven.).

(16) Después, más al tiempo, busqué otro trabajo. Trabajé en un supermercado. Me fue chévere porque aprendí un poquito de todo, ¿no?, si trabajaba por fuera, como si trabajaba por dentro (CREA, Ven.).

No obstante, la mayoría de los empleos de indicativo después de como si se da en los textos de ficción (31: CORPES XXI 27, CREA 1, CREA. Version anotada 3) destacando otra vez España (14) y la zona de México / Centroamérica (13: El Salvador 6, México 3, Nicaragua 3 y Guatemala 1). En Argentina, Uruguay, Colombia y Chile fueron registrados en total 4 casos. Como hemos advertido supra, en los textos de ficción la construcción aparece, en primer lugar en las novelas y en los relatos:

(17) - Pues que se bañe con nosotros - me dijo como si era la cosa más natural del mundo (CORPES XXI, Nic.).

(18) Ella se acercó un poco y yo traté de hablar bajo, como si iba a contarle algún chisme (CORPES XXI, Guat.).

Los ejemplos pertenecientes a los géneros de no ficción son minoritarios habiendo sido documentados solo 13 casos repartidos entre CORPES XXI (8), CREA (3) y CREA. Versión anotada (2). La mayoría de los empleos se dio en España (3) y la zona de México / Centroamérica (4: El Salvador 1, México 1, Nicaragua 1 y Guatemala 1). El resto de las formas se ha registrado en la zona del Río de la Plata (3: Argentina 2, Uruguay 1) Venezuela (2) y Bolivia (1). Salvo tres casos del discurso oral comentados supra, los demás ejemplos proceden de textos periodísticos tales como reportajes, artículos, etc.: 
(19) Un hombre hizo como si hacía cola para presuntamente depositar o realizar algún pago en dicha agencia; otro ingresó y dijo que le urgía dialogar con el gerente del lugar y cuando lo tenía cara a cara, desenfundó su arma de fuego y amenazó (CREA. Versión anotada, Guat.).

(20) La nueva Canasta no utiliza mini canastas estacionales para frutas y verduras y vestimenta, como si hacía la anterior, dada la mayor disponibilidad de estos artículos durante todo el año (CREA. Versión anotada, Ur.).

Cabe señalar que si en el ejemplo (19) la forma hacía sustituye hiciera / hiciese, no lo hace en el (20) donde hacía al acompañar el adverbio afirmativo sí, no reemplaza formas subjuntivas mencionadas supra, lo que confirma una vez más que el factor clave para la interpretación de los datos del presente análisis es la repartición sintáctico-semántica de la construcción objeto de este estudio.

\section{LA REPARTICIÓN SINTÁCTICO-SEMÁNTICA}

Como hemos apuntado, de acuerdo con los corpus de la RAE, las mayores cantidades de la construcción en cuestión se observan en España (17) y en la zona México / Centroamérica (17: El Salvador 7, México 4, Nicaragua 4, Guatemala 2). En 6 países restantes se han documentado solo 10 casos, es decir, menos de dos por país.

No obstante, las distribuciones sintáctico-semánticas en España y en México / Centroamérica son bastante diferentes. Tenemos que ver con dos opciones distintas. La fórmula de la primera es como $s i+\{$ cantaba $=$ (por) SUBJ $\}$, es decir, la forma de copretérito sustituye las formas subjuntivas cantara / cantase. En cambio, la segunda corresponde a la fórmula como $s i+\{$ cantaba $\neq$ (no por) SUBJ $\}$, lo que quiere decir que el copretérito no es equivalente funcional del SUBJ. La diferencia entre dichas opciones se ve motivada por factores sintáctico-semánticos.

El elemento de enlace de la fórmula como $s i+\{c a n t a b a=$ (por) SUBJ $\}$ es unión de dos conjunciones subordinantes como y si que forman el nexo como si que funciona como exponente introductor de las cláusulas con valor de comparación y contrafactualidad.

Por su parte, en la fórmula como $s i+\{$ cantaba $\neq$ (no por) SUBJ $\}$ la unión de las cláusulas se lleva a cabo mediante cuatro nexos diferentes:

1. Como si: conjunción subordinante como + conjunción subordinante $s i$.

2. Como sí: conjunción subordinante como + adverbio afirmativo sí.

3. Cómo si: adverbio interrogativo cómo + conjunción subordinante si.

4. Cómo sí: adverbio interrogativo cómo + adverbio afirmativo sí.

Además, siendo conjunción subordinante, como aporta a menudo el matiz de comparación en las estructuras de tipo tanto si... como si o tal / tan... como si (véase también supra el párrafo 2). 
Con respecto a las diferencias entre España y México / Centroamérica, cabe subrayar que la distribución sintáctico-semántica de la variedad peninsular casi en todos los casos (14 de 17) corresponde a la fórmula como si $+\{$ cantaba $\neq$ (no por) $\mathrm{SUBJ}\}$, lo que significa que solo en 3 ejemplos se lleva a cabo el modelo como $s i+\{$ cantaba $=$ (por) SUBJ $\}$ :

$\underline{\text { como } s i+\{c a n t a b a \neq \text { (no por) } \mathrm{SUBJ}\}}$

(21) Podría parecer que juega, como si hacía Aznar, a provocar la guerra de nervios... (CORPES XXI, Esp.).

(22) Al final era un grupo de médicos o cirujanos, los escuadrones de Dios, les llamaban, que en ocasiones consideraban la adecuación del paciente con criterios como si era alcohólico, por ejemplo (CORPES XXI, Esp.).

(23) En ambos casos, tanto si era para recuperar el tiempo perdido, como si era para contar con los dedos los acentos del Himno a la memoria, o a la memoria de Mnemosyne, el enterramiento era parte substancial de la obra (CREA, Esp.).

$\underline{c o m o s i}+\{$ cantaba $=$ (por) $\mathrm{SUBJ}\}$

(24) Dios escribe derecho en renglones torcidos, y por mí como si era analfabeto, me daba igual (CORPES XXI, Esp.).

(25) - Gracias - le dije poniendo un gesto festivo que servía tanto para el caso de que fuera verdad como si era una broma (CORPES XXI, Esp.).

De modo que, aunque es en España donde se da la mayor cantidad de los empleos de la construcción como si + cantaba (17), son muy pocas las ocasiones cuando cantaba sustituye las formas subjuntivas cantara / cantase (3). En la mayoría de los casos cantaba aparece con valor comparativo-afirmativo después de como sí o se da en las estructuras de comparación introducidas por las expresiones adverbiales de tipo tanto... como, construcciones donde el empleo de las formas cantara / cantase no suele darse.

En cambio, los ejemplos documentados en la zona de México / Centroamérica (17) corresponden, básicamente al modelo como si $+\{$ cantaba $=$ (por) SUBJ $\}$ (13 casos: El Salvador 7, Nicaragua 4, Guatemala 2) siendo los exponentes de la fórmula como $s i+\{$ cantaba $\neq$ (no por) $\mathrm{SUBJ}\}$, claramente, minoritarios (solo 4 casos, todos de México):

como $s i+\{$ cantaba $=$ (por) $\mathrm{SUBJ}\}$

(26) Y enseguida como si estaba dando un mensaje en el mismo seno de las Naciones Unidades, continuó diciendo (CORPES XXI, El Salv.).

(27) Estoy cansada y me duele el pescuezo porque casi me desprendés la cabeza con el frenazo que metiste sólo porque venías hecha una fiera, como si era mi culpa que nos detuviera la policía (CORPES XXI, Nic.).

(28) Tiempo después, Mike repitió la escena, fue a catedral, bajó las escaleras del edificio y caminó hacia el sótano de catedral, era curioso, la tumba de San 
Romero, estaba vacía, le hicieron una escultura en bronce y el balazo se le miraba como si era de verdad, una verdadera obra maestra (CORPES XXI, El Salv.).

$\underline{\text { como } s i+\{\text { cantaba } \neq \text { (no por) } \mathrm{SUBJ}\}}$

(29) En marzo de 2006, transcurridos seis meses de la reunión con el asesor, se planteaba la entrega de avances, enfrentándose a la poca posibilidad de terminar la tesis y finalmente a la suspensión de la misma: “¿Cómo sí podía con la secundaria [el servicio social], la universidad, con comida, y todo?, y no sé si fue como una depresión, porque no concebía la idea de que no me estaba dando tiempo" (CORPES XXI, Méx.).

(30) Arcadia: Ya ve como sí era un difunto, comadre (CORPES XXI, Méx.).

Como se ve, al comparar España con la zona de México / Centroamérica, resulta evidente que las soluciones son totalmente opuestas: en la Península casi todos los casos (14 de 17) representan el modelo como $s i+\{$ cantaba $\neq$ (no por) SUBJ $\}$, mientras que en la región hispanoamericana en cuestión la mayoría de los ejemplos corresponde a la fórmula como $s i+\{$ cantaba $=$ (por) SUBJ $\}$ (13 de 17). En otras palabras, mientras que en la zona México / Centroamérica cantaba realmente en la mayoría de los casos del corpus reemplaza las formas subjuntivas cantara y cantase, en España tales sustituciones son poco frecuentes.

\section{OBSERVACIONES FINALES}

Recapitulando las informaciones presentadas supra cabe subrayar que la difusión del fenómeno en cuestión se ve bastante limitada en todos los niveles.

Así, como hemos señalado, el empleo de cantaba después de como si (como sí, cómo si, cómo si) tiene lugar en el caso de 8 verbos (del total de 20) concentrándose de hecho en los verbos haber (15), hacer (7), ser (13) (35 ejemplos del total de 44; un $80 \%$ ). Con 12 verbos restantes no se registró ningún ejemplo de cantaba con como si.

Desde el punto de vista de la repartición diatópica predominan con gran ventaja dos zonas: España (17) y México y Centroamérica (17: El Salvador 7, México 4, Nicaragua 4, Guatemala 2) que constituyen un 75\% del total (34 ejemplos del total de 44). El tercer lugar lo ocupa, a gran distancia, la zona del Río de la Plata con 5 formas (Argentina 3 y Uruguay 2).

Con respecto a la repartición diafásica, cabe subrayar que la mayoría de los empleos de cantaba después de como si se ha documentado en los textos de ficción (31 del total de 44) destacando España (14) y la zona de México / Centroamérica (13: El Salvador 6, México 3, Nicaragua 3 y Guatemala 1). En Argentina, Uruguay, Colombia y Chile fueron registrados solo 4 casos. Los empleos de no ficción fueron documentados solo en 13 casos. La mayoría se dio en España (3) y en la zona de 
México / Centroamérica (4: El Salvador 1, México 1, Nicaragua 1 y Guatemala 1). Las demás formas han sido registradas en la zona del Río de la Plata (3: Argentina 2, Uruguay 1) Venezuela (2) y Bolivia (1). Salvo tres casos del discurso oral comentados supra (uno grabado en la radio española, aunque el hablante, al parecer, era boliviano), todos los ejemplos proceden de textos periodísticos tales como reportajes, artículos, etc.

En cuanto a la repartición sintáctico-semántica, resulta que las zonas mayoritarias de España y México / Centroamérica al optar por soluciones opuestas, demuestran importantes diferencias. Así, en la Península casi todos los casos (14 de 17) representan el modelo como $s i+\{$ cantaba $\neq$ (no por) $\mathrm{SUBJ}\}$, lo que quiere decir que cantaba, de hecho, mantiene su valor indicativo y no suele sustituir las formas subjuntivas cantara y cantase. En cambio, en la zona de México / Centroamérica la mayoría de los ejemplos corresponde a la fórmula como $s i+\{$ cantaba $=$ (por) $\mathrm{SUBJ}\}$ (13 de 17), lo que significa que cantaba en la mayoría de los casos del corpus realmente reemplaza las formas cantara y cantase. De modo que podemos deducir que la tendencia hacia la sustitución del subjuntivo por el indicativo se manifiesta más en Hispanoamérica (en primer lugar en la zona de México / Centroamérica) que en España donde en la mayoría de los casos cantaba se emplea con valor comparativo-afirmativo después de como sí o aparece en las estructuras de comparación introducidas por las expresiones adverbiales de tipo tanto si... como si o tal / tan... como si (cfr. supra, los ejemplos 12, 13, 21, 22, 23).

Aun así, no se puede excluir del todo la paulatina evolución hacia la consolidación del uso de cantaba después de como si. Pues, como es sabido, en las lenguas románicas hay dos posibilidades de modalización del contenido proposicional: una mediante el modo subjuntivo y otra, a través del desplazamiento temporal de los tiempos de indicativo (véase, a este respecto, p. ej., Nowikow 2013 o 2017b: 19-20). El empleo de cantaba después de como si representa este segundo recurso. De hecho, es lo que pasa en algunas lenguas románicas, por ejemplo en francés, donde comme si se combina con los tiempos del modo indicativo (Il me traite comme si j'étais son servant). El español opta, generalmente, por el subjuntivo, aunque algunos nexos que incluyen la conjunción subordinante como, por ejemplo, como que o como cuando, suelen construirse con el modo indicativo (cfr., p. ej., Alarcos Llorach 1994: 363 y Pamies Bertrán, Nowikow 2015: 96).

Sin embargo, de momento la frecuencia del empleo de cantaba después de como $s i$ es bajísima. Por ejemplo, como demuestran los datos estadístico-numéricos proporcionados por el CORPES XXI, la llamada frecuencia normalizada de 35 ejemplos de 7 verbos calculada a base de cinco distribuciones (zona, país, período, tema, tipología) oscila entre el 0,00 0,04 casos por millón, lo que quiere decir que el cambio distribucional en cuestión todavía está muy lejos de su posible realización. 


\section{BIBLIOGRAFÍA}

Alarcos Llorach, E. (1994).Gramática de la Lengua Española. Madrid: Editorial Espasa Calpe, S.A. Gutiérrez Araus, M.L. (2004). Problemas fundamentales de la gramática del español como2/L. Madrid: Arco/Libros. S.L.

Montolío, E. (1999). Las construcciones condicionales. In I. Bosque, V. Demonte (eds.), Gramática descriptiva de la Lengua Española, vol. III (pp. 3643-3737). Madrid: Real Academia Española/Espasa-Calpe.

NGLE: Nueva gramática de la lengua española (2010). Real Academia Española/Asociación de Academias de la Lengua Española, Morfología/Sintaxis I, segunda tirada corregida. Madrid: Espasa Libros, SLU.

Nowikow, W. (2013). Sobre la modalización del contenido proposicional: contraste tipológico entre lenguas románicas y eslavas. In A.Pamies Bertrán (ed.) (pp. 65-72). Granada: Editorial Comares.

Nowikow, W. (2017a). Tiempos verbales. In W. Nowikow (ed.), Gramática contrastiva españolpolaco (pp. 127-178). Colección “Manufactura Hispánica Lodziense”, 2. Łódź: Wydawnictwo Uniwersytetu Łódzkiego.

Nowikow, W. (2017b). Modos verbales. In W.Nowikow (ed.), Gramática contrastiva españolpolaco (pp. 11-126). Colección "Manufactura Hispánica Lodziense”, 2. Łódź: Wydawnictwo Uniwersytetu Łódzkiego.

Pamies Bertrán, A., Nowikow, W. (2015). Los modos verbales en español y en polaco. Colección "Manufactura Hispánica Lodziense", 1. Łódź: Wydawnictwo Uniwersytetu Łódzkiego.

Pérez Saldanya, M. (1999). El modo en las subordinadas relativas y adverbiales. In I. Bosque, V. Demonte (eds.), Gramática descriptiva de la Lengua Española, vol. II (pp. 3253-3322). Madrid: Real Academia Española/Espasa-Calpe.

REAL ACADEMIA ESPAÑOLA: Banco de datos (CORPES XXI) [en línea]. Corpus del Español del Siglo XXI (CORPES), <http://www.rae.es> [fecha de la consulta: 24.11.2018].

REAL ACADEMIA ESPAÑOLA: Banco de datos (CREA) [en línea]. Corpus de referencia del español actual, <http://www.rae.es> [fecha de la consulta: 29.11.2018].

REAL ACADEMIA ESPAÑOLA: Banco de datos (CREA. Versión anotada) [en línea]. Corpus de referencia del español actual, <http://www.rae.es> [fecha de la consulta: 29.11.2018].

Veiga, A., Mosteiro Louzao, M. (2006). El modo verbal en cláusulas condicionales, causales, consecutivas, concesivas, finales y adverbiales de lugar, tiempo y modo. Salamanca: Ediciones Universidad de Salamanca. 